\title{
Postures and motion shaping musical experience
}

Chapter for the Routledge Companion to Embodied Music Interaction, v. March 2016

Rolf Inge Godøy

\begin{abstract}
Sound-producing body motion and associated body postures shape musical sound in interaction with musical instruments or the vocal apparatus, making images of such body motion and postures integral to our experiences of music. In this chapter we shall look at some soundproducing body motion features, and focus on how postures at salient moments in time serve as landmarks for both motion and sound, as well as how the fusion of small-scale motion units and sounds by so-called coarticulation creates posture-centered sound-motion objects in musical experience.
\end{abstract}

\section{Introduction}

We may typically experience music as a continuous stream of sound, and as is one of the basic tenets of this book, also as a continuous stream of body motion sensations in parallel with the sound. As such, continuity is a basic feature of musical experience, yet most of us would agree that we also perceive a series of distinct events in music: within the stream of music, we perceive singular sounds, tones, chords, often fused into singular melodic, harmonic, rhythmic, and textural patterns, often in synchrony with singular sound-producing and/or sound-accompanying body motion events. In other words, we also have discontinuity in musical experience with such singular events. The coexistence of continuity and discontinuity in music is what we shall try to better understand in this chapter by considering how postures and motion shape musical experience.

The term posture is here used to designate the shape and position of any soundproducing parts of the body, also known in the human motion literature as effectors, i.e. the vocal apparatus, the fingers, hands, arms, shoulders, torso, etc., in interaction with an instrument (e.g. the shape and position of fingers and hands on a keyboard). We also have various kinds of postures in sound-accompanying motion, like in 
dancing, walking, gesticulating, or any other kind of body motion to music. As for the term motion, it designates any continuous displacement and/or change in posture, i.e. the continuous transition of effectors from one shape and/or position to another shape and/or position (e.g. the fingers/hands moving from a widely spaced chord at the extremities of the keyboard to a densely spaced chord at the center of the keyboard). Postures at certain salient points in the music, such as at downbeats and other accents, are considered particularly significant, and are called key-postures. Key-postures are intermittent, meaning discontinuous with regards to motion, whereas the intervening motion between postures is continuous.

It is this coexistence of discontinuity and continuity as the interplay of postures and motion that we shall study in this chapter. With this focus in mind, we shall first have a look at what we call ecological constraints on music making, followed by some elements of motor cognition and considerations of different timescales of sound and motion in music, before presentations of the main concepts of posture and motion. These concepts then come together in what we call sound-motion objects in music, and the perception and cognition of these objects attest to intermittency as an integral element of musical experience, an element that is manifest in the crucial role of key-postures in musical experience.

\section{Ecological constraints}

Assuming that musical sound (at least traditionally) is made by human body motion in interaction with real-world physical instruments or the human vocal apparatus, there are obviously a number of constraints at work in this sound-production. These constraints extend from the physics of the instrument or physiology of the vocal apparatus (and also include the physics of the sound-producing environment, i.e. room acoustics) to the sound-producing body motion, and thus also include a number of biomechanical and neurocognitive constraints. These may collectively be called ecological constraints, and in particular the following constraints of the instrument or voice are relevant for our considerations of postures and motion:

- Traditional musical sounds afford distinct instrumental modes of excitation, such as hitting, stroking, shaking, bowing, etc., something that in turn constrain possible motion (e.g. we usually do not shake a trumpet or blow on a maracas). 
- Also, instruments afford distinct duration-dependent types of excitatory motion: sustained (continuous motion such as in bowing or blowing), impulsive (abrupt motion such as in hitting or kicking), and iterative (rapid back-and-forth motion such as in a trill or tremolo), as opposed to electronic instruments which do not have such inherent constraints categories in that they have an unbounded energy source, i.e. they are not dependent on actions that have a beginning and an end.

- Instruments afford modes of modification (modulation of sound), which likewise reflect delimited motion chunks by the performer, such as in the making of a vibrato, in the opening-closing a mute, in back-and-forth tremolo motion, etc.

- The sound output of an instrument will in most cases have a certain temporal coherence (within certain limits) due to energy dissipation, i.e. although the duration and the length of the decay of a sound may vary, there will usually still be some minimal feature of continuity in most instrumental or vocal sounds.

- There is a basic perceived instrument identity, what we could call instrument coherence, across different registers (low-high) and different modes of playing, in spite of rather drastic physical spectral differences between the output sounds.

- There may be overlap between sounds due to incomplete damping (and room reverb) in cases of multiple excitations (e.g. repeated hitting of a cymbal), resulting in a contextual smearing of the sound that is actually an element of coarticulation, i.e. the fusion of otherwise separate motion units into larger units (Godøy, 2014).

And as for sound-producing body motion, we typically have the following constraints on the motion of the effectors:

- Biomechanical limitations on maximal speed, amplitude, and force, and the need for rests and posture changes to avoid fatigue and/or strain injury.

- Instantaneous displacement of the effectors is not possible, as all motion takes time, and hence, there will always be gradual transitions between postures.

- There is usually a need for anticipatory positioning of effectors, e.g. hands need to be placed in a favorable position for finger motion on the keyboard before the moment of key depression, something that is the hallmark of coarticulation.

- Notably so, coarticulation works both ways in time, so there is also a so-called carryover effect of recently performed motion on presently performed motion, in addition to the mentioned anticipatory motion (Godøy, 2014). 
- Effectors are (variably so) connected anatomically, e.g. fingers on a hand are not totally independent of each other, hence there will often be co-activations and socalled spatial coarticulation, so that motion in one effector may recruit motion in one or more neighboring effectors, in some cases even whole torso motion for maximal force or expression.

- Some sequences of effector motion are easy, others are difficult or even impossible, and this goes for most instruments, such as for finger motion on keyboards and string instruments (e.g. speed limits on single finger displacement whereas multiple finger motion can be very fast), or tongue motion in vocal articulation (e.g. the difficulty of rapid $t i-t i-t i-t i$ vs. the ease of rapid $t i-k u-t i-k u$ ).

- There are so-called phase-transitions due to constraints, i.e. switch of effector use with speed and amplitude changes (Haken, Kelso, \& Bunz, 1985), such as the transition from individual finger motion to a continuous underarm rotating motion when performing an acceleration from slow to a fast tremolo between two tones on a keyboard.

- Anticipatory cognition is not only a biomechanical necessity, as implied by the phenomenon of coarticulation, but also a necessity of motor control because of latency in the control process. The phenomenon of the so-called psychological refractory period suggests that human reaction times may be too slow for continuous, feedback-based, so-called closed loop control, hence the need for planning and initiating motion chunks in advance, and hence also the idea of action gestalts (Klapp \& Jagacinski, 2011).

- The principle of intermittency, i.e. of discontinuity, in motor control is a way to optimize motor control by piecewise pre-programming and open loop control of motion, so that continuous feedback control is not needed (Loram, van De Kamp, Lakie, Gollee, \& Gawthrop, 2014).

- As for the distribution of effort by the muscles over time in human body motion, there seems to be indications of intermittency in so-called ballistic motion, e.g. in hitting, kicking and rapid stroking, whereas we do not know enough about the distribution of effort in more continuous motion such as continuous bowing or blowing (however, we suspect that there might be more effort in the initial stage of sustained motion than towards the end). 
All in all, it seems reasonable to conclude that there is constraint-based discontinuity in human motor control (and probably also in effort), and that the mentioned intermittency in motor control (and again, probably also in effort) may be seen as optimal for human motion, something that in turn will fit well with the posturemotion duality presented in this chapter.

\section{Motor cognition}

Although we may group music-related body motion into the two main categories of sound-producing and sound-accompanying (Godøy \& Leman, 2010), there will be similarities between these categories in view of energy envelope shapes and timing, e.g. similarities in amplitude of body motion and sound, and similarities in rhythmic patterns of body motion and sound, etc. Also, music-related body motion may often be multifunctional: a musician's body motion may be directly sound-producing (e.g. lifting a hand to hit a chord), yet also communicative towards other musicians in an ensemble (the same hand motion as an upbeat cue) and/or towards the audience (the same hand motion as a show of affect). This means that we usually have a rich set of music-related body motion contributing to our perception of musical sound.

The idea that images of body motion contribute to our perception of sound is known as the motor theory of perception. The motor theory of perception, including variants of this theory, claim that images of body motion are evoked by another sense modality, in our case eminently so by auditory sensations. This means that when we hear sound, we also tend to imagine body motion we believe has generated the sound. Initially proposed in linguistics (Liberman \& Mattingly, 1985), it has later been extended to other domains such auditory perception in general (Galantucci, Fowler, \& Turvey, 2006), and the visual domain (Berthoz, 1997). Similar motor theory perspectives have been applied to our experience of the world in general (Gallese \& Metzinger, 2003), including most areas of everyday life (Jeannerod, 2006), and social cognition (Wilson \& Knoblich, 2005). These and similar publications suggest that images of motion are at the core of our cognitive and affective constitution.

It thus seems reasonable to believe that there is mental simulation of postures and motion going on in musical experience, what could be called motormimetic cognition (Godøy, 2001; 2003). We have in our own research looked at what is called air instruments, i.e. listeners' spontaneous renderings of assumed sound-producing body motion (Godøy, Haga, \& Jensenius, 2006) and other spontaneous, dance-like body 
motion to musical sound, including what we have called sound-tracing, i.e. listeners' spontaneous renderings of motion features associated with sound features (Nymoen, Godøy, Jensenius, \& Torresen, 2013). Also related studies such as (Küssner, Tidhar, Prior, \& Leech-Wilkinson, 2014) seem to suggest that there are several links between different sound features and sensations of motion in musical experience.

In a motor theory perspective, the perceived musical sound is then spontaneously associated with assumed sound-producing body motion, also in cases where there are no performers to be seen. Although the extent and vividness of this motor imagery in music listening remains to be further explored, we hypothesize that motor images in music also include images of postures and motion, as is the main tenet of this chapter.

\section{Timescales of sound and motion}

Clearly, we have concurrent elements at different timescales in music, ranging from that of single vibrations in the sub-millisecond duration range to that of tones and phrases in the several seconds duration range, and beyond that, to sections and whole works in the range of several minutes and even hours. When considering postures and motion in music, we thus need to be more specific about the different timescales involved.

Comparing timescales of sound and motion, there are obviously speed limitations on human body motion. However, there are also similarities at some timescales as we can see in the following overview that we have found useful in our research on musicrelated body motion:

- Micro: continuous sound and motion with features such as pitch, loudness, stationary timbral features, and fast transients and fluctuations in the sound.

- Meso: typically in the duration range of 0.5 to 5 seconds, including dynamic, pitch-related, and timbre-related envelopes, as well as shorter melodic, rhythmic, and textural patterns, what could be called quantal elements in music (Godøy, 2013).

- Macro: the duration range of sections, movements, and whole works, consisting of many meso timescale chunks in succession.

The way we understand the term posture, it is typically associated with the meso timescale, i.e. that postures occur intermittently at the rate of very approximately 
somewhere between 0.5 to 5 seconds, in most cases probably in the shorter range of this meso timescale.

As for motion, there are constraints at work here between the different categories: impulsive sound-motion events may change into a series of sustained sound-motion events with increased duration (i.e. going from a series of staccato onsets to a series of overlapping sustained sounds), and into a fused iterative event with decreased duration (i.e. going from a series of staccato onsets to a continuous tremolo), hence undergo qualitative changes, so-called phase-transitions (Haken, Kelso, \& Bunz, 1985).

The principle of constraint-based sound-motion categories at different timescales seems to be quite well founded, however we need research to identify more precisely the different thresholds for factors such as duration and density of events, amplitude and velocity of motion, and the derivatives of velocity (acceleration and also jerk) in musical performance, in particular in view of the emergence of the mentioned phenomena of key-postures and coarticulation. Put differently: what is the typical duration threshold for the manifestation of key-postures? And: what is the typical duration threshold for the manifestation of coarticulation, i.e. for the fusion of otherwise separate motion and sound events into some larger and strongly coherent chunk?

\section{Postures}

The fundamental tenet of this chapter is that music-related body motion is centered on postures at salient moments in time. This is based on human motion control research that point to the hierarchical and goal-directed nature of human body motion (see Grafton and Hamilton (2007) for a lucid summary of relevant past and present research). Specifically, there is research that supports the idea of posture-centered body motion, both with respect to prior planning and to control during the actual unfolding of the motion. Adapting the ideas of key-frames and interframes from cartoon animation, and thus distinguishing between key-frames at salient moments in time and the continuous or gradual transition between these key-frames by the socalled interframes, Rosenbaum, Cohen, Weiss, and van der Wel (2007) suggested we may apply a similar distinction between key-frames and continuous motion in human motor control. 
In our own research, we have chosen to use the term key-posture rather than keyframes, to emphasize the shape aspect of the effectors. The essential idea of this keyposture + motion model is that planning and control is much more efficient with such a division than it would be with a continuous feedback model, i.e. that intermittent control is more efficient than continuous control. We have adapted this key-posture + motion model so that key-postures are to be found at salient moments in time, typically at downbeats and other accented points in the music. Furthermore, we believe there are motion trajectories to and from these key-postures, what we call respectively prefixes and suffixes to the key-postures.

Although we may subjectively pick out and recall such key-postures in our experiences of music, similarly to how listeners spontaneously detect the beat in musical sound, documenting in more detail what are key-postures in a continuous stream of motion and sound is quite challenging, resembling the challenges of machine-based beat tracking in music. Although threshold values for quantity of motion have been suggested as cues for relative stationary postures (Hogan \& Sternad, 2007), it may be difficult to reliably pick out stationary postures from musicians' motion capture (or other) data, because effectors are hardly ever still. What seems to be a promising strategy is to look at the velocity data of musicians' motion, as there seems to be fairly clear velocity peaks at moments of downbeat and other accents: like in a collision, when the fingers/hands hit the bottom of the keys on a keyboard or the mallet/hand impacts on a percussion instrument, there is an immediately ensuing upward rebound, i.e. a velocity reversal, and we hypothesize that this collision and the immediately following velocity reversal is the point of the downbeat, hence also the point of the key-posture (Godøy, 2013; 2014). We may then in turn interpret the motion capture data at this point in time as that of a key-posture, e.g. the hand's shape at that point in time in relation to a keyboard, or the mallet-handarm-shoulder shape at that point in time in relation to a drum membrane.

Key-postures serve as orientation points for musical features such as dynamical, rhythmical and textural patterns: we reason that e.g. a key-posture may be located at the "1" (downbeat point) at the bottom of a rapid downward motion (velocity peak) in a $3 / 4$ waltz pattern, followed by subsequent "2" and "3" in an upward motion. Such a key-posture may also coincide with the harmonic root and hence a tonal orientation point, i.e. the bass tones and chords defining the tonal center of the music in the actual context (see Godøy (2013) and (2014) for some examples). But also with regards to 
aesthetic and affective significations, we suspect key-postures play an important role in the experience of musical performance (similar to facial expression), as may be seen in the famous caricatures of Liszt in Figure 1.

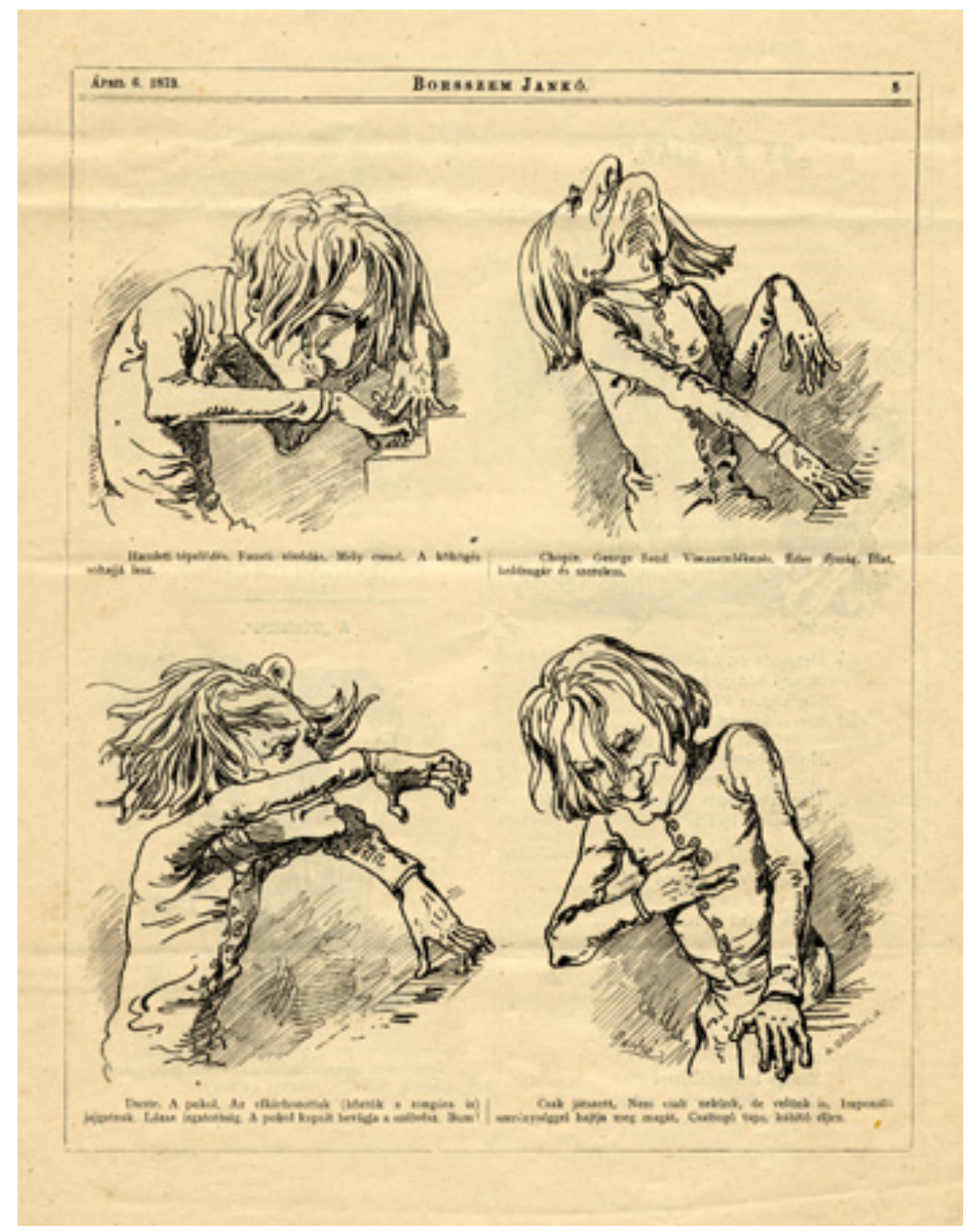

Figure 1. Caricature of Franz Liszt's postures by A. Göschl (Göschl 1873).

\section{Motion}

Motion in our context typically includes the transition from one posture to another. In this transition, instantaneous displacement is impossible, and instead, we end up with some continuous transition and what we could call 'smearing' by coarticulation of the otherwise more distinct postures. This is why coarticulation is defined as the fusion of otherwise distinct motion and/or sound events into higher level motion and/or sound units, as e.g. in the fusion of finger motions and tones in rapid succession to an 
arpeggio figure or an ornament, conceived and perceived as coherent units, as gestalts in both motion and sound.

Coarticulation has been extensively studied in linguistics (Hardcastle \& Hewlett, 1999), and to a certain extent also in other domains of human motion (Rosenbaum, 2009), but not so much in music (Godøy, 2014). From our perspective of musicrelated body motion, musical interpretation and performance may be understood as a matter of transforming what may initially be conceived as a series of discrete pitch events into a fused and coarticulated motion chunk, meaning that the transition from Western common practice notation symbols to a real performance is actually a process of coarticulatory transformation. In this process, the key-postures remain the orientation points of the motion, hence, the idea of posture-centered sound-motion objects in the next section.

Furthermore, motion comprises a number of features, such as shape, range (or amplitude), speed and direction, taken together as velocity, as well as the second and third derivatives, i.e. acceleration and jerk (Hogan \& Sternad, 2007), all of which are highly significant for the overall affective and aesthetic manifestation of the music.

Additionally, there are of course also a large number of motion features at different levels of resolution, i.e. from coarse, overall, whole body motion down to the tiniest nuance of various kinds of expressive motion. Following Pierre Schaeffer and co-workers (Schaeffer, 1966; Chion, 1983), we could say that most features of musical sound may actually be correlated with motion shapes, e.g. that all the features of Schaeffer's so-called morphology of the sonic objects such as various timbral fluctuations, could be extended to also include posture shapes for the quasi-stationary features of musical sound.

\section{Sound-motion objects}

Extending Schaeffer's notion of sonic objects to include motion, we can here see a convergence of the elements presented above in what we call sound-motion objects (Godøy, 2006). The crucial element here is that sound and motion are considered fused into coherent units, typically in the meso timescale range, conceived and perceived holistically, and as will be argued, centered on key-postures.

The main point of Schaeffer's method was a top-down differentiation of sonic object features, starting with the overall dynamic, pitch and timbre-related shapes (the so-called typology), and then progressively differentiating more and more sub- 
features down to the tiniest fluctuation in any sonic object (the abovementioned morphology). With our extension of this to also include motion shapes, we could say that there is a general amodal shape cognition at work here, as was one of the main points of so-called morphodynamical theory (Thom, 1983; Petitot, 1985).

Variants of object cognition have also been the focus of other research, however the cumulative and/or holistic nature of auditory objects seems still not well understood, although there has been significant progress in the direction of recognizing cross-modal elements at work in auditory object perception (Griffiths \& Warren, 2004; Bizley \& Cohen, 2013). Also, we need more research along the lines of Grossberg and Myers (2000) of understanding how continuous auditory streams may be kept in memory as more of 'solid' and 'all-at-once' kinds of auditory objects.

As for the holistic nature of motion objects, it has been convincingly argued that human behavior is organized as a series of action gestalts, and one reason for this being that reaction times are constrained by the abovementioned psychological refractory period (Klapp \& Jagacinski, 2011). Related to this is the suggestion that also highly complex polyrhythmic chunks may be conceived as one 'simple' unit, facilitating its realization (Klapp, Nelson, \& Jagacinski, 1998)

In recognizing the convergence of various arguments in favor of what we could call posture-centered sound-motion perception and cognition in music, we should also understand this as based on the principle of intermittency in human motor control, and by extension, also in human perception.

\section{Intermittency in musical experience}

It can be argued that there is an element of intermittency in music-related body motion in the form of key-postures, both for the sake of ergonomics and for the sake of orientation, c.f. the arguments for posture-oriented motion referred to above.

In the domain of human motor control, there has been a long-lasting debate as to the status of the mentioned closed loop and open loop control schemes (Elliott, Helsen, \& Chua, 2001). A closed loop scheme means that there is continuous sensory feedback (from vision, hearing, touch, etc.) so that the motion of the effectors may by continuously corrected (similar to a thermostat continuously sensing the temperature and continuously adjusting the energy flow to a stove), whereas an open loop scheme means no feedback in the course of the motion, hence that all control has to be done prior to the motion, e.g. as in hitting a golf ball. Although the closed loop scheme may 
seem plausible when we observe the high level of detail motor control in human behavior, it has also been objected that closed loop control in many cases may be too slow, and hence unrealistic, in demanding activities such as sports and musical performance.

Recent research suggests that intermittent control may be disguised as continuous control in that a series of intermittent control impulses that are very close in time may result in apparent continuous motion (Loram et al. 2014). Put another way, we have piecewise continuous motion between the key-postures, analogous to the continuous curves in so-called $B$-splines in mathematics emerging from a small set of discrete values in a polynomial.

In other words, the key-posture-by-key-posture model is in our opinion a useful way to understand this intermittency, and we may generate testable hypotheses to this effect, suggesting a long-term research program ahead of us here. The advantage of such an approach will be first of all that of shedding light on the enigmatic relationship of continuity and discontinuity in musical experience, a relationship that has intrigued thinkers since Husserl's work on time perception at the end of the nineteenth century (Husserl, 1991) (see Godøy (2010) and (2011) for discussions). Second, the advantage will be accommodating a number of constraints on human motion and perception as listed above. Third, by shifting the main focus of the chunk to the key-posture, it could also be suggested that postures are primordial for the establishment of sound-motion objects. Fourth, the idea of intermittency could shed light on the notion of beat, suggesting making 'beat' a sub-category of the more general principle of intermittency in musical experience, concretely the temporal moment of the key-posture (and probably also of maximum effort). But notably so, intermittency is not equal to perfect periodicity, hence an intermittency understanding of 'beat' would also apply to asymmetrical and even aperiodic rhythmical patterns, as long as there is a discontinuity in motor control and effort.

\section{Conclusion}

Musical interaction is based on a number of constraints, and pointing this out is not to say that music is hampered or restrained, but rather that musical interaction carries with it some significant features from the physics of musical instruments and from human body motion. Specifically, the organization of human motion by way of keypostures seems relevant in musical contexts because it fits well with the chunking of 
musical streams into coherent units, into what we have called sound-motion objects. The model of intermittent key-postures interleaved with continuous motion certainly needs more research, however, it has the attractive feature of reconciling sensations of continuity and discontinuity in musical experience.

Ahead of us is an extensive work of modeling, testing, and documenting musical performance as the transformation from score to a series of key-postures connected by coarticulated motion trajectories, a transformation resulting in coherent sound-motion objects. Also we need to study how this applies to perception, where we shall look for a reverse identification of intermittent key-postures and coarticulated trajectories from the continuous sound-motion flow. Lastly, we hope to demonstrate applications in music analysis, music classification, and music information retrieval, of how various styles, genres, and modes of musical interaction correlate with distinct music-related postures and body motion patterns.

\section{References}

Berthoz, A. (1997). Le sense du mouvement. Paris: Odile Jacob.

Bizley, J. K., \& Cohen, Y. E. (2013). The what, where and how of auditory-object perception. Nature Reviews Neuroscience, 14, 693-707.

Chion, M. (1983). Guide des objets sonores. Paris: INA/GRM Buchet/Chastel.

Elliott, D., Helsen, W., \& Chua, R. (2001). A century later: Woodworth's (1899) twocomponent model of goal-directed aiming. Psychological Bulletin, 127(3), 342357.

Galantucci, B., Fowler, C. A., \& Turvey, M. T. (2006). The motor theory of speech perception reviewed. Psychonomic Bulletin \& Review, 13 (3), 361-377.

Gallese, V., \& Metzinger, T. (2003). Motor ontology: The Representational Reality Of Goals, Actions And Selves. Philosophical Psychology, 16(3), 365-388.

Grafton, S. T., \& Hamilton, A. F. (2007). Evidence for a distributed hierarchy of action representation in the brain. Human Movement Science, 26, 590-616.

Griffiths, T. D., \& Warren, J. D. (2004). What is an auditory object? Nature Reviews Neuroscience, 5(11), 887-92.

Godøy, R. I. (2001). Imagined Action, Excitation, and Resonance. In R. I. Godøy \& H. Jorgensen (Eds.), Musical Imagery (pp. 237-250). Lisse: Swets \& Zeitlinger. Godøy, R. I. (2003). Motor-Mimetic Music Cognition. Leonardo, 36(4), 317-319. 
Godøy, R. I. (2006). Gestural-Sonorous Objects: embodied extensions of Schaeffer's conceptual apparatus. Organised Sound, 11(2), 149-157.

Godøy, R. I. (2010). Thinking Now-Points in Music-Related Movement. In R. Bader, C. Neuhaus and U. Morgenstern (Eds.) Concepts, Experiments, and Fieldwork. Studies in Systematic Musicology and Ethnomusicology (pp. 245-260). Frankfurt am Main: Peter Lang.

Godøy, R. I. (2011). Sound-action awareness in music. In D. Clarke, \& E. Clarke (Eds.) Music and Consciousness (pp. 231-243). Oxford: Oxford University Press.

Godøy, R. I. (2014). Understanding Coarticulation in Musical Experience. In M. Aramaki, M. Derrien, R. Kronland-Martinet \& S. Ystad (Eds.): Sound, music, and motion. Lecture Notes in Computer Science (pp. 535-547). Berlin, Heidelberg: Springer-Verlag.

Godøy, R. I., Haga, E., \& Jensenius, A. R. (2006). Playing ‘Air Instruments’: mimicry of sound-producing gestures by novices and experts. In S. Gibet, N. Courty, \& J.F. Kamp (Eds.), GW2005, LNAI 3881 (pp. 256-267). Berlin, Heidelberg: Springer-Verlag.

Godøy, R. I. and Leman, M. (Eds.) (2010). Musical Gestures: Sound, Movement, and Meaning. New York: Routledge.

Grossberg, S., \& Myers, C. W. (2000). The resonant dynamics of speech perception: Interword integration and duration-dependent backward effects. Psychological Review, 107(4), 735-767.

Göschl, A. (1873). Caricatures of Liszt, Borsszem Jankó, 6 April 1873, p. 5.

Haken, H., Kelso, J., \& Bunz, H. (1985). A theoretical model of phase transitions in human hand movements. Biological Cybernetics, 51(5), 347-56.

Hardcastle, W. J. \& Hewlett, N. (Eds.) (1999). Coarticulation: theory, data and techniques. Cambridge: Cambridge University Press.

Hogan, N., \& Sternad, D. (2007). On rhythmic and discrete movements: reflections, definitions and implications for motor control. Experimental Brain Research, 181, $13-30$.

Husserl, E. (1991). On the Phenomenology of the Consciousness of Internal Time, 1893 -1917. English translation by John Barnett Brough. Doredrecht-BostonLondon: Kluwer Academic Publishers.

Jeannerod, M. (2006). Motor cognition. Oxford: Oxford University Press. 
Klapp, S. T., \& Jagacinski, R. J. (2011). Gestalt Principles in the Control of Motor Action. Psychological Bulletin, 137(3), 443-462.

Klapp, S. T., Nelson, J. M., \& Jagacinski, R. J. (1998). Can People Tap Concurrent Bimanual Rhythms Independently? Journal of Motor Behavior, 30(4), 301-322.

Küssner, M. B., Tidhar, D., Prior, H. M., \& Leech-Wilkinson, D. (2014). Musicians are more consistent: Gestural cross-modal mappings of pitch, loudness and tempo in real-time. Frontiers in Psychology, 5, Article 789. doi:

10.3389/fpsyg.2014.00789

Liberman, A. M., \& Mattingly, I. G. (1985). The Motor Theory of Speech Perception Revised. Cognition, 21, 1-36.

Loram, I. D., van De Kamp, C., Lakie, M., Gollee, H., \& Gawthrop, P. J. (2014). Does the motor system need intermittent control? Exercise and Sport Science Review, 42(3), 117-125.

Nymoen, K., Godøy, R. I., Jensenius, A. R., \& Torresen, J. (2013). Analyzing correspondence between sound objects and body motion. ACM Transactions on Applied Perception, 10(2).

Petitot, J. (1985). Morphogenèse du Sens I. Paris: Presses Universitaires de France.

Rosenbaum, D. (2009). Human Motor Control (2 ${ }^{\text {nd }}$ edition). San Diego: Academic Press.

Rosenbaum, D., Cohen, R. G., Jax, S. A., Weiss, D. J., \& van der Wel, R. (2007). The problem of serial order in behavior: Lashley's legacy. Human Movement Science, 26(4), 525-554.

Schaeffer, P. (1966). Traité des objets musicaux. Paris: Éditions du Seuil.

Thom, R. (1983). Paraboles et catastrophes. Paris: Flammarion.

Wilson, M. \& Knoblich, G. (2005). The case for motor involvement in perceiving conspecifics. Psychological Bulletin, 131(3), 460-473. 